\title{
Opportunities and Challenges for Precision Personalized Therapy in Multiple Myeloma
}

\author{
Nahla AM Hamed* \\ Professor of Clinical Hematology, Egypt
}

*Corresponding author: Nahla AM Hamed, Professor of Clinical Hematology, Faculty of Medicine, Alexandria University, Egypt

\section{ARTICLE INFO}

Received: 慧 December 03, 2019

Published: December 10, 2019

Citation: Nahla AM Hamed. Opportunities and Challenges for Precision Personalized Therapy in Multiple Myeloma. Biomed J Sci \& Tech Res 23(4)-2019. BJSTR. MS.ID.003945.

\section{ABSTRACT}

Treatment of MM is administered empirically based on recurrence risk rather than genetic events. Each MM patient deserves the most effective, data driven therapeutic regimen tailored to his case. Choosing the optimal initial therapy of MM remains a challenge. The implementation of promising precision medicine strategies seems to be closer than ever.

Abbreviations: MM: Multiple Myeloma; scRNA seq: Single-Cell RNA Sequencing; CTCs: Circulating Tumor Cells; NGS: Next-Generation Sequencing

\section{Introduction}

The main goal of precision medicine is to match each patient with the right drug to his case [1]. Precision medicine in oncology relies on identifying the key biomarkers deriving cancer progression [2]. Current approaches in precision oncology aim at matching specific DNA mutations rather than the tumor histology or tumor type to drugs. However, incorporation of genome-wide RNA profiles has not yet been clinically assessed [3]. Multiple myeloma (MM) is a malignancy of terminally differentiated plasma cells with a median survival of 6 years [3]. MM has wide and remarkable heterogeneity of the mutational spectrum across patients and a complex sub clonal structure [3]. MM is increasingly being recognized as more than one disease, characterized by marked cytogenetic, molecular and proliferative heterogeneity [4].

There are two key aspects of MM pathogenesis: the genetic lesions intrinsic to the malignant clone and the interaction between myeloma cells and the microenvironment of the BM [4]. Hallmarks of MM include chromosomal translocations and copy number alterations [3]. MM is classified as either non hyperdiploid or hyperdiploid. The hyperdiploid subtype (50-60\% of MM patients) is characterized by presence of copy number alterations such as trisomies of the odd chromosomes. Non hyperdiploid subtypes harbor translocations between 14q32, immunoglobulin heavy chain locus and one of several partners oncogenes including MAF (16q23), MAFB (20q11), FGFR3/MMSET (4p16.3), CCND1(11q13), and CCND3 (6p21) [4].Heterogeneous abnormalities in DNA methylation, non-coding RNA expression, chromatin modifications and mutations in epigenetic regulators among others have been also described in MM (5).The paracrine interaction between MM and its microenvironment involve secretion and uptake of soluble factors (chemokines, cytokines and growth factors) as well as exosomes (exocytotic nanovesicles) that harbor oncogenic mRNA, miRNA and proteins [5].However, the causal drivers of MM pathogenesis are still unclear [3].

Choosing the optimal initial therapy of MM remains a challenge [4]. Treatment is administered empirically on the basis of recurrence risk rather than genetic events [3]. There are currently at least five classes of active agents available for myeloma treatment: alkylating agents, anthracyclines, corticosteroids, immunomodulatory drugs and proteasome inhibitors. There are also many potential agents including monoclonal antibodies, drug-conjugated antibodies, drugs targeted to molecular abnormalities, microRNA inhibitors or mimics and immune therapies such as chimeric antigen receptors $\mathrm{T}$ cells and anti-PD1 agents [4]. Despite recent therapeutic advancements, relapse remains mostly inevitable [3]. Malignant plasma cells still have mechanistic avenues by which they can evade even the most promising new therapies [5]. The disease is fatal in the majority of patients [3]. 
Which Patients Are Appropriate for Precision Medicine?

Novel precision medicine combinations are designed to develop for high risk MM patients with rapidly progressed disease in spite of standard of care therapies [6]. In resistant MM patients, precision medicine prevents deploy of certain drugs to minimize the use of ineffective therapy and avoid attendant toxicities [6]. The timely identification of treatment options in a personalized manner for relapsed MM patients is a major challenge [3]. Adopting precision medicine into routine clinical practice has the potential to radically change the natural history of MM by identifying biomarkers that predict efficacy or resistance within an individual's sub clonally heterogeneous tumor [5].

\section{Potential Candidates for Precision Medicine Trials in MM are}

Vemurafenib (BRAF inhibitor) produces durable response in relapsed refractory BRAF V600E-positive myeloma. Trametinib (MEK inhibitor) experienced at least 25\% reduction of measurable MM protein in RAS-mutated MM [6].1q amplified MM is among the most common copy number alterations encountered, with a confirmed adverse effect on survival. Patients with $1 \mathrm{q}$ amplified MM might benefit from genetically tailored treatment involving cell cycle and ubiquitin inhibitors or a combination thereof [7]. Similarly, Novel FGFR inhibitors targeting FGFR-3 mutated tumors as well as the identification of activating alterations in IDH1/2 and NTRK1/2/3 may be interesting as potential candidates for precision medicine trials in MM [6].

\section{Challenges for Precision Medicine Approaches in MM}

\section{Clonal Heterogeneity in MM}

MM is characterized by branching pattern of clonal evolution in which distinct sub clonal tumor population may evolve by selective pressure of therapy [3]. Dynamic intra-patient (sub clonal heterogeneity) has been described recently in myeloma. Presence of distinct subclones at alternating points along longitudinal sampling (clonal tides) suggests that they may be persistent below detectable levels within a patient. Cases of high-risk disease may harbor exceptional sub-clonal heterogeneity that may be accelerated by DNA damaging therapies [5].Therapy targeting molecular alterations present in only a subset of myeloma cells might not achieve clinical benefit or may even allow more rapid growth of subclones lacking the relevant genomic target [6].

\section{Environment-Mediated Drug Resistance (EM-DR):}

The bone marrow microenvironment comprises immune cells and multiple other cell types as well as the extracellular matrix (ECM), a non-cellular component comprising a collection of proteins [8]. The relationship between MM and its microenvironment is bidirectional. Crosstalk between MM cells and their microenvironment converge on critical proliferation / survival pathways that can directly affect chemotherapeutic efficacy and propagates resistance to therapy by agonizing prosurvival / anti-apoptotic mechanisms [5]. Several genes of BM stromal cells (BMSC) have major functions in RNA processing (44 genes), cell cycle regulation (53 genes), and ubiquitin proteasome pathway activation (35 genes) when cocultured with myeloma cell lines [4]. Profiling the microenvironment by scRNAseq and/ or mass cytometry which allows simultaneous measurement of up to 50 markers at single cell resolution may better inform targeted therapy. However, scRNAseq requires the use of specialized tools which are still in their development stage [3].

\section{Challenges in Molecular Diagnostic Tests}

Bone marrow aspirates in MM have limited ability to fully represent the genetic complexity of the disease as well as its geographic diversity due to focal sampling bias. Liquid biopsies to sample peripheral blood and quantify circulating tumor cells (CTCs) and cell free/ circulating tumor DNA [5] have emerged as a promising tool in MM but further refinements are needed to perfect liquid technologies [6].

\section{The Time Required for Sequencing}

The average turnaround time required from sample collection to sequencing data was 6 weeks. However, most relapsing MM patients experience rapid progression and need immediate treatment. Implementing NGS based recommendations in a realworld setting needs using rapid run sequencing which provides results in a few days. This may be more expensive initially but may become cost effective with greater use [3].

\section{Drug- Specific Resistance Biomarkers}

No one molecular marker, genotype or mechanism is universally responsible for conferring resistance to any specific therapy in MM [5]. Precision medicine MM drugs will require appropriate companion diagnostic tests [6]. There is a pressing need to integrate drug- specific resistance biomarkers with several very promising emerging quantitative molecular profiling technologies [5]. The MSk-IMPACT ${ }^{\mathrm{TM}}$ and Foundation One $\mathrm{CDx}^{\mathrm{TM}}$ which has recently approved by FDA for clinical sequencing are often not fully optimal for MM. Robust clinical assays must be able to detect functional consequences such as pathway activation rather than simply molecular alterations in specific members of cellular networks [6].

\section{Other Challenges}

The need for clinical trials to evaluate the security and efficacy of these targeted therapies as well as to validate predictive biomarkers that may allow an appropriate best candidate selection and improvement of myeloma patients' survival and quality of life [9].Immune profiling will be essential as precision medicine of the future in MM will involve precisely matching patients with the appropriate combinations of targeted and/or immune-based therapies [6]. 


\section{Emerging Methods in Clinical Profiling of MM}

Single Cell Transcriptomic Technology (Targeted/ qPCR or

RNA seq): The first published use of scRNAseq in MM was able to distinguish MM CTCs from healthy plasma cells and demonstrated that single-CTC transcriptomes were representative of bulk bone marrow sample transcriptomes [5].

\section{Conclusion}

These data suggest that the use of a personalized therapeutic approach in MM is likely to improve the outcomes for newly diagnosed, resistant or relapsed myeloma in the future.

\section{Acknowledgement}

None.

\section{Conflict of Interest}

No conflict of interest.

\section{References}

1. Gonzalez-Calle V, Keane N, Braggio E, Fonseca R (2017) Precision medicine in myeloma: challenges in defining an actionable approach. Clinical Lymphoma Myeloma and Leukemia 17(10): 621-630.

ISSN: 2574-1241

DOI: $10.26717 /$ BJSTR.2019.23.003945

Nahla AM Hamed. Biomed J Sci \& Tech Res

CC (P) This work is licensed under Creative

Submission Link: https://biomedres.us/submit-manuscript.php
2. Savard MF, Khan O, Hunt KK, Verma S (2019) Redrawing the lines. The next generation of treatment in metastatic breast cancer. ASCO Educational Book: e8.

3. Lagana A, Beno I, Melnekoff D, Leshchenko V, Madduri D, et al. (2018) Precision medicine for relapsed multiple myeloma on the basis of an integrative multiomics approach. JCO Precis Oncol.

4. Yang WC, Lin SF, Su YC (2018) Multiple Myeloma: Personalized medicine based on pathogenesis. EMJ 3(2): 78-89.

5. Harding T, Baughn L, kumar S, Ness BV (2019) The future of myeloma precision medicine: integrating the compendium of known drug resistance mechanisms with emerging tumor profiling technologies. Leukemia 33(4): 863-883.

6. Auclair D, Lonial S, Anderson C, kumar Sk (2019) Precision medicine in multiple myeloma: are we there yet? Expert Review of precision medicine and drug development 4(2): 51-53.

7. Sklavenitis-Pistofidis R, Reidy M, Huynh D, Salem KZ, Park J, et al. (2018) Founding precision therapy in 1q-amplified multiple myeloma. Blood 132: 1007.

8. Sklavenitis-Pistofidis R, Bustoros M, Ghobrial IM (2019) Bone marrow niche in multiple myeloma and its precursor state. HemaSphere Educational Updates in Hematology Book 3(S2): 121-123.

9. Gonzalez-Calle V, Fonseca R (2017) Towards precision medicine in myeloma. Medicina (B Aires) 77(3): 222-226.

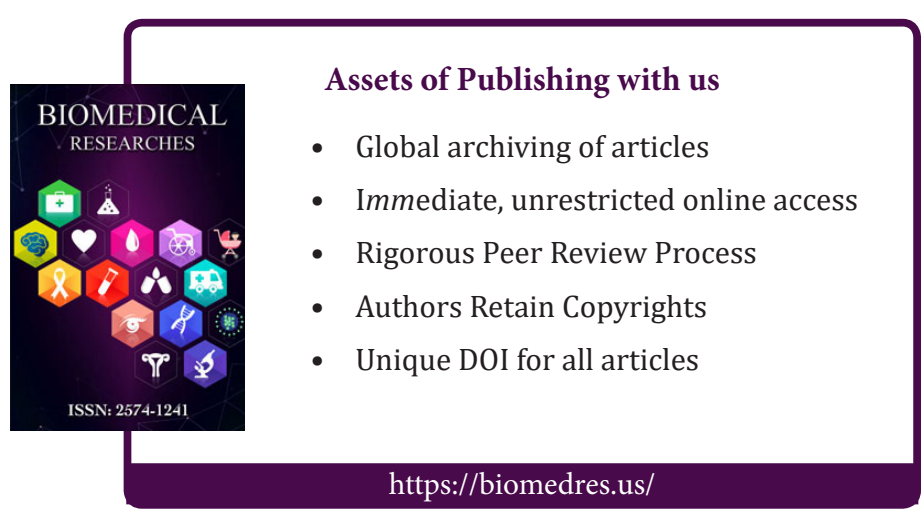

